\title{
Dosimetric Evaluation of a Treatment Planning System Using the Aapm Medical Physics Practice Guideline 5.a (MPPG 5.a) Validation Tests
}

Yousif A Yousif ( $\square$ Yousif.Yousif@health.nsw.gov.au )

North West Cancer Centre https://orcid.org/0000-0002-1640-0346

Jackson Zifodya

North West Cancer centre, Tamworth Hospital

\section{Research Article}

Keywords: Dosimetric evaluation, quality assurance, treatment planning system, MPPG 5.a

Posted Date: December 14th, 2021

DOI: https://doi.org/10.21203/rs.3.rs-1020530/v1

License: (c) (i) This work is licensed under a Creative Commons Attribution 4.0 International License.

Read Full License 


\section{Abstract}

In this work, the AAPM Medical Physics Practice Guideline 5.a (MPPG 5.a) validation tests package was used to evaluate the dosimetric performance of a new version of the Eclipse treatment planning system (TPS) algorithms. A series of tests were developed and comparisons between TPS calculated and corresponding beam data measurements have been performed for basic beam validation, heterogeneity correction and IMRT/VMAT dose validation tests. Measurements were performed using a Varian IX Linear Accelerator with the $6 \mathrm{MV}, 6 \mathrm{MV}$ FFF and $18 \mathrm{MV}$ photon beams, and 6, 9, 12, 16, $20 \mathrm{MeV}$ electron beams. Results for basic dose validation tests yielded differences within $3 \%$ for all point doses and pass rate greater than $95 \%$ for all depth profiles using $3 \% / 3 \mathrm{~mm}$ criteria. For testing the ability of the TPS in accounting for tissue inhomogeneity, corresponding comparisons were performed with the presence of a heterogeneous media to simulate an air inhomogeneity. Results showed a mean deviation between the TPS calculated and measured of $1.9 \%$, reaching a maximum of $2.8 \%$ for the AAA algorithm. For IMRT/VMAT validation tests, our local criteria passing rate of $95 \%$ was used, but no consensus of the tolerance exists. Our results agree well with the data reported accuracy in previous studies of Eclipse TPS. In summary, the AAPM MPPG 5.a validation tests are a valuable package for evaluating dose calculation accuracy and are very useful for TPS upgrade checks, commissioning tests and routine TPS QA.

\section{Introduction}

A treatment planning system (TPS) is considered an essential component of external beam radiation therapy. It is used to generate beam shapes and dose distributions with the intent to maximize tumour dose and minimize normal tissue complications. To achieve this goal, a dose coverage to a tumour volume should be within the $+7 \%$ to $-5 \%$ range [1]. In general, treatment planning is a complex process that involves many steps and relies on many factors to achieve the required accuracy, and therefore, a standard and stringent Quality Assurance (QA) program of the TPS has to be implemented. Several guidance documents were published to help medical physicists in developing tests for commissioning and validation of TPS [2-4]. The AAPM TG-53 [2] and the IAEA TECDOC-430 published comprehensive reports on commissioning and QA of a computerized TPS for radiotherapy [3]. Recently, the AAPM published the Medical Physics Practice Guideline 5.a (MPPG 5.a), which provides concise guidelines on the commissioning and QA of TPS dose calculations algorithms [5]. Although these previous reports can be considered as complete [2-4] and are strongly encouraged for the implementation of a robust and comprehensive QA program, their drawback is associated with the need for lots of resources and time to be implemented effectively which can be difficult to achieve in a limited-resources department. Even though the MPPG 5.a sets minimum requirements for commissioning and QA of TPS dose calculations, it has also offered the clinical physicists the flexibility in data acquisition using a range of detectors and tools. The validation process described by the MPPG5.a is divided into four sections; basic photon beams, photon beam heterogeneity corrections, IMRT/VMAT validation, and electron beam validation. These are sections 5 to 8 , respectively, of the report [5]. Although many groups have published their experience in commissioning and verifying different TPS algorithms [6-9], a few references are found on 
implementation of the MPPG 5.a validation tests $[10,11]$. In the present work, the evaluation of the accuracy of Eclipse TPS (Varian Medical Systems, Palo Alto, CA) calculation algorithms is performed comparing the measured values with the calculated one. The MPPG5.a validation tests are implemented with different organization of the original validation suits of validation tests and are made as follows: A) the basic photon and electron tests are merged into one section. The heterogeneity corrections for both photon and electron are also combined into one section (B); and Section C) is IMRT/VMAT dose validation tests. The goal of this work was to evaluate the dosimetric accuracy of the newer version of the Eclipse TPS dose calculation algorithms. To this aim, the AAPM MPPG 5.a validation test packages has been used.

\section{Materials And Methods}

\section{A Treatment planning System}

The Eclipse TPS Version 15.6.05, was used for all calculations in this study. The dose calculation algorithms employed by the TPS were Anisotropic Analytical Algorithm (AAA) and Acuros XB algorithm (AXB) for photon beam and the Electron Monte Carlo (eMC) for electron beam. Dose calculations were performed using a virtual water tank (a cube of water created in the Eclipse TPS) for most basic validation tests. For heterogeneity correction tests, plans were made in a heterogonous phantom in TPS, and a number of points were added to the plan representing the location of the ionization chamber. For IMRT/VMAT dose validation tests, clinical reference plans were re-calculated in ArcCHECK's virtual phantom and exported to the machine for delivery. All plans were generated in the TPS for the MPPG 5.a validation tests in a single test patient. For basic validation tests, each plan contained beams with the same field size but different energies. All dose calculations were performed using a grid size of a $2 \mathrm{~mm}$ and exported separately for each beam for comparisons.

\section{B Measurement equipment and technique}

Beam data and point doses were acquired following the MPPG 5.a's recommendations using various detectors in a scanning water tank, solid water phantoms, or a 3D detector array device. Table 1 summarises the MPPG 5.a tests with an associated description alongside the equipment used. The radiation source was a Varian iX linear accelerator (Linac) equipped with a Millennium 120 multi-leaf collimator (MLC). The Linac delivers photon beams of 6 and $18 \mathrm{MV}$ (with flattening filter), $6 \mathrm{FFF}$ (flattening filter-free), and electron beams of 6, 9, 12, 16 and 20 MeV. A PTW 3D MP3-M water tank (PTW, Freiburg, Germany) was used with the following PTW detectors; Semiflex ionisation chamber (active volume of $0.125 \mathrm{~cm}^{3}$ ), a 3D Pinpoint ionization chamber (active volume of $0.016 \mathrm{~cm}^{3}$ ), and an Electron diode (active volume of $0.03 \mathrm{~cm}^{3}$ ) for dosimetric measurements with the acquired data processed using PTW's MEPHYSTO software version 3.4 (PTW, Freiburg, Germany). Following the TRS-398 protocol for reference dosimetry [12], a PTW Farmer and Roos Parallel Plate chambers were used with a PTW UNIDOS electrometer. For heterogeneity measurements, custom made phantoms were designed from solid water 
slabs (Gammex-RMI, Middleton, WI, USA) and Styrofoam to mimic lung tissue as shown in Fig 2. A schematic of phantoms dimensions, all with $10 \mathrm{~cm}$ backscatter after the Styrofoam is illustrated Fig 3; (a) photon heterogeneity dose measurements, (b) $6 \mathrm{MeV}$ electron dose measurements; (c) $9 \mathrm{MeV}$ electron dose measurements; and (d) for 12-20 MeV electron dose measurements. Furthermore, a Gammex electron density CT phantom (Gammex RMI, Middelton, WI, USA) was also used to establish the CT-to-ED curve, and the Sun Nuclear 3D ArcCHECK diode array (Sun Nuclear, Melbourne, FL, USA) was used for IMRT/VMAT verification. All phantoms were scanned on a Phillips Big Bore computed tomography (CT) scanner (Philips Healthcare, Amsterdam, Netherlands) with a 2- mm slice thickness, and then CT datasets were exported to the TPS for dose calculations

\section{C The MPPG 5.a validation tests}

\section{C. 1 Basic beam validation}

The basic photon and electron beam algorithm validation tests $5.1-5.3$ are essential tests to verify the agreement of the dose distribution in the planning module with the commissioning data, reference clinical calibration conditions, beam profiles and output factors for large and small field sizes. In test 5.1, dose distributions of a $40 \times 40 \mathrm{~cm}^{2}$ field incident on a large water phantom at an SSD of $100 \mathrm{~cm}$ were compared to the corresponding commissioning profiles. Test 5.2; photon and electron absolute dose calculation, under reference conditions, were also performed; a field size of $10 \times 10 \mathrm{~cm}^{2}$ at an SSD of 100 $\mathrm{cm}$, at reference depth were compared to the measured dose per $\mathrm{MU}$ at the same reference conditions. Test 5.3, measured percentage depth doses (PDD), beam profiles, and output factors for square field sizes of $2,3,4,5,7,10,20$, and $40 \mathrm{~cm}^{2}$ were compared to calculated data.

Furthermore, the MPPG 5.a recommends six additional basic validation tests for photon beams, five with static MLC field shapes with the jaws placed at clinically relevant positions (5.4-5.8) and one with a nonphysical wedge (5.9). For electron beams, two validation tests, 8.1 and 8.2, were performed in a homogenous water phantom. Test 8.1 used a custom cut-out shape in a $15 \times 15$ applicator. This field represents a clinically relevant shaped field. Test 8.2 aims to verify the surface irregularities obliquity using a standard $10 \times 10$ applicator at an oblique beam incidence of $20^{\circ}$. Fig 1 illustrates the Beam's Eye View of the MLC fields created in the TPS. Test 5.4, a small MLC-shape, was intended to mimic a small MLC defined treatment field. A large MLC-shaped field with extensive blocking (e.g., mantle shape) was used in test 5.5. Test 5.6 is an off-axis field with the maximum allowed leaf over-travel in the X-direction with the $Y$ direction defined by the jaws. Test 5.7 is an irregular MLC field shape. Test 5.8 is an MLC defined field at an oblique incident angle of $20^{\circ}$ from the vertical. Test 5.9 verifies a large field with an enhanced dynamic wedge (EDW) measured using an ArcCHECK diode array. A $20 \times 20 \mathrm{~cm}^{2}$ EDW field with wedge angles of $15^{\circ}, 30^{\circ}, 45^{\circ}$ and $60^{\circ}$ were measured for 6 and $18 \mathrm{MV}$ photon beams. For this test, two sets of measurements were performed, one with $\mathrm{Y} 1-\mathrm{IN}$ wedge orientation and the other with $\mathrm{Y} 2$ OUT wedge orientation. 
All beam profile and PDD measurements for basic validation tests $(5.4-5.9,8.1-8.2)$ were obtained in a 3D scanning water tank. For photons, one PDD and two beam profiles at dmax, $10 \mathrm{~cm}$ or $25 \mathrm{~cm}$ depth were measured in the inplane and crossplane directions. For electrons, one PDD and one inplane and one crossplane profiles were measured at a depth of the $90 \%$ isodose. All scanning beam data were compared to the calculated data from Eclipse TPS using an open-source scanning data analysis tool ScanDoseMatch (http://www.qxrayconsulting.com/sdm/) [7, 13]. Gamma analysis was performed using a gamma criterion of a $2 \%$ / $2 \mathrm{~mm}$ following the MPPG 5.a recommendations. Besides the scanned data, additional verification point doses were also acquired at $1.5 \mathrm{~cm}$ and $10 \mathrm{~cm}$ depths along the central axis (CAX) for tests 5.4, 5.5, 5.7 and 5.8. The absolute dose at each point of the measured profile was acquired using a calibrated Pinpoint chamber.

\section{C. 2 Heterogeneity correction validation}

The minimum recommended validation tests include confirming the CT-to-ED curve (test 6.1) and verifying TPS calculations beyond heterogeneities (tests $6.2 \& 8.3$ ). The CT-to-ED curve was verified in the TPS by scanning an electron density phantom and confirming the CT values of various inserts were reported correctly in the TPS. For dose verification beyond the heterogeneous media for photon beams (test 6.2), measurements were taken at two points located along the CAX; one upper and one lower placed

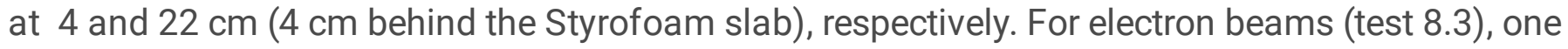
measurement was collected close to dmax in heterogeneity phantom, and at least two measurements were acquired along the CAX in the dose fall-off region. Measurements near dmax were taken at $2.5 \mathrm{~cm}$ and $3.5 \mathrm{~cm}$ for 6 and $9 \mathrm{MeV}$ and at $6.5 \mathrm{~cm}$ for 12,16 and $20 \mathrm{MeV}$. Measurements along the dose fall-off region were taken at 3 and $3.5 \mathrm{~cm}$ for $6 \mathrm{MeV}, 4$ and $4.5 \mathrm{~cm}$ for $9 \mathrm{MeV}, 9$ and $10 \mathrm{~cm}$ for $12 \mathrm{MeV}, 7$ and 10 $\mathrm{cm}$ for $16 \mathrm{MeV}$, and 7 and $11 \mathrm{~cm}$ for $20 \mathrm{MeV}$. All measurements were compared to the Eclipse TPS points representing the location of the ionization chamber.

\section{C. 3 IMRT/VMAT dose validation}

Several validation tests are recommended for IMRT/VMAT delivery modality. Tests 7.1 and 7.2 are for a very small MLC defined shape field. The PDD and output factor for a $2 \times 2 \mathrm{~cm}^{2}$ MLC-shape field were measured in a scanning water tank with a PTW E Diode. For tests 7.3 and 7.4, twelve cases; two IMRT and ten VMAT plans for prostate, endometrium, pelvis, head and neck, brain, and rectum treatment sites, were used. All plans were re-calculated onto the ArcCHECK verification phantom, delivered and evaluated using gamma criteria of $3 \% / 3 \mathrm{~mm}$ and $2 \% / 2 \mathrm{~mm}$ with absolute dose, global normalization, and a low dose threshold of $10 \%$. Point dose measurements were also performed using a Pinpoint ionization chamber inserted into the ArcCHECK. Finally, for test 7.5, the MPPG 5.a recommends an external review as a complete end-to-end test for IMRT/VMAT delivery validation. Therefore, to verify the accuracy of the TPS, an end-to-end test was performed using the Australian Clinical Dosimetry Service (ACDS) Audit 
phantoms [14-16] The ACDS audit plans were created, delivered and analyzed following the ACDS Audit specifications.

\section{Results}

The results of the MPPG 5.a validation for the Eclipse photon and electron beam models created for our Varian iX Linac are presented below.

\section{A Basic validation tests}

A comparison between some of the measured and TPS calculated beam data for tests 5.1 and 5.3 are shown in Fig 4. This includes crossplane profile of a $6 \mathrm{MV} 40 \times 40 \mathrm{~cm}^{2}$ field, PDD of a $18 \mathrm{MV} 40 \times 40$ $\mathrm{cm}^{2}$, crossplane profile of a $6 \mathrm{MVFFF} 10 \times 10 \mathrm{~cm}^{2}$ field, and output factors for a $6 \mathrm{MV}$ photon beam. The results showed good agreement between measured and calculated beam data where gamma values were less than 1.0 for most PDDs and profiles. In addition, all calculated output factors for the field sizes were within $2 \%$ of the measured values.

Fig 5 (a-g) displays the results from comparisons of the measured and calculated profiles for tests 5.45.9 , and $8.1 \& 8.2$ with corresponding gamma criteria of $2 \%$ and $2 \mathrm{~mm}$. The results revealed good agreement between the measured and calculated profiles, with all points passing the gamma index values of 1.0. In some circumstances, gamma index values above 1.0 were recorded around the tail of the beam profile for large field sizes. Point dose verifications were performed for both photon algorithms, AAA and AXB. Two point doses were evaluated for each of the three photon energies (42 points in total). Table 2 shows the results obtained for the differences between measured and calculated point doses for AAA and AXB. The results revealed that most of the measured data agreed well with the TPS calculated data for all the tests and were within the $2 \%$ tolerance recommended by the MPPG 5.a.

\section{B Heterogeneity correction validation}

A comparison between the CT numbers from the Eclipse generated CT-to-ED curve and those from the scanned electron density CT phantom showed good agreement and the maximum observed difference was $12 \mathrm{HU}$ as shown in table 3. For heterogeneity verifications, comparisons between measurements and TPS calculated point doses distal to the heterogeneous media are shown in table 4. All calculated point doses are within $3 \%$ of the measurements for heterogeneous media for both photon algorithms and all three different energies. Results for electron energies are tabulated in table 5; the differences between the measured and TPS calculated point doses for all electron energies were slightly high, average and maximum differences observed were $5 \%$ and $7 \%$, but were however within the $7 \%$ tolerance recommended by the MPPG 5.a. 


\section{C IMRT/VMAT dose validation}

For the IMRT/VMAT dose validation tests, the MPPG 5.a recommends relatively tight evaluation criteria, however there is no widely accepted criteria published for small fields or IMRT/VMAT. All tests were evaluated using 3\% / 3mm and 2\% / 2mm Gamma criteria. For Test 7.1, the PDD and output were evaluated for very small field size, and the measured PDD agreed with the TPS calculated PDD within $2 \%$ beyond the build-up region for all beams. Fig 6.a and 6.b show an example of gamma analysis between measured and calculated data a PDD and crossplane profile for a $2 \times 2 \mathrm{~cm}^{2}$ field size. For test 7.2 , the output was measured using a diode detector and a Pinpoint chamber, and the dose comparisons were within 2\%. For test 7.3-7.4, Eclipse TPS calculated dose distributions were compared against the ArcCHECK measurements on two IMRT and ten VMAT plans for prostate, endometrium, pelvis, head and neck, brain, and rectum treatment sites. The results of the Gamma passing rate using criteria of $3 \% / 3 \mathrm{~mm}$ and $2 \% / 2 \mathrm{~mm}$ are tabulated in table 6 together with the number of fields/arcs. The average passing rates across all sites using criteria of $3 \% / 3 \mathrm{~mm}$ and $2 \% / 2 \mathrm{~mm}$ were $98.8 \%$ and $97.7 \%$, respectively, while the average absolute point dose difference was $1 \%$. For Test 7.5 , the ACDS audit plans passing rate was over $95 \%$ for gamma criteria of $3 \% / 3 \mathrm{~mm}$.

\section{Discussion}

Verification of the accuracy of the dose calculation algorithms is considered an important step in the radiotherapy treatment process. This ensures that the TPS calculated dose meets the prescribed dose and reduces the discrepancies between the TPS estimated dose and the actual dose delivered to the patient [17]. In this work, the AAPM MPPG 5.a validation tests were used to evaluate the accuracy of a new version of the Eclipse TPS algorithms. The MPPG 5.a validation tests were re-arranged in slightly different sections, in three instead of the four sections in the original guideline's recommendations.

In the first section, tests 5.1 and 5.3 are considered confirmation tests and are straightforward in which they do not require further measurement apart from commissioning data. Results show good agreements between the TPS calculated data and measurements. The commissioning data for all field sizes and energies are within the recommended specifications by MPPG 5.a. Test 5.2 verifies the photon and electron beams absolute output under reference calibration condition in the TPS. The results for the photon beam confirm the agreement with measurements and are within the $0.5 \%$ tolerance as recommended by MPPG 5.a for photon beams. Results for the electron beam are within $1 \%$. While the MPPG 5.a does not have specific recommendations for tolerance for the electron beam in this test, the tolerance selected in this work was from the AAPM TG-142. For the static photon and electron validation tests (5.4-5.9 and 8.1-8.2), comparisons between TPS calculated and measured PDDs and profiles were confirmed using the ScanDoseMatch analysis software $[5,7,13]$. Almost all the tests analyzed yielded gamma index values below 1.0 using a $2 \% / 2 \mathrm{~mm}$ passing criteria (relative mode) for photons and electrons (Fig 5). For all point dose, the agreement between calculation and measurements were less than or equal to $2 \%$ (table 2). Nevertheless, results have also indicated some disagreements where gamma 
index was greater than 1.0 for some large profiles at the penumbra regions, and these results support previous findings [11].

In the second section, the heterogeneity correction tests; the accuracy of the CT-to-ED conversion curve is confirmed in tests 6.1 (table 3 ). For the heterogeneity point dose measurements for photon beam, the Eclipse AAA and AXB algorithms results fall within the tolerance of $3 \%$ for the two field sizes and three energies applied in this test (Table 4). For electron validation test 8.3, the Eclipse eMC algorithm, point dose results were higher, with a maximum difference of $6.8 \%$ from the measurements. Though these results were relatively high, they still easily met the 7\% recommended tolerance by MPPG 5.a (Table 5). These results are in agreement with the findings of the previous results $[5,7,11]$.

In the IMRT/VMAT dose validation section, for tests 7.1 and 7.2, the results for the PDD and profiles scored a passing rate greater than $95 \%$ using $2 \% / 2 \mathrm{~mm}$ criteria for all energies investigated. The output factor for the small field was within $2 \%$ of the calculated TPS data. Tests 7.3-7.4 were considered as most import tests as these verified the beam model accuracy for the Eclipse TPS. All the IMRT and VMAT calculations were only performed using the AXB algorithm as this is what is clinically used in our centre. The results for all IMRT/VMAT cases, the minimum pass rate was $95.6 \%$ and $94.3 \%$ for $3 \% / 3 \mathrm{~mm}$ and $2 \% / 2 \mathrm{~mm}$ criteria, respectively. The average and standard deviation in point dose was $0.9 \pm 1 \%$. In our clinic, the minimum pass rate is set at $95 \%$ for criteria of $3 \% / 3 \mathrm{~mm}$, so based on our local criteria all cases passed. For test 7.5, we followed ARPANSA's ACDS end-to-end verification format for external audits and this gave us confidence in our TPS validation and modelling.

\section{Conclusion}

The aim of this study was to evaluate the performance of a new version of the Eclipse TPS algorithms, AAA, AXB and eMC using MPPG 5.a. The results obtained demonstrated the accuracy of the Eclipse version 15.6 TPS algorithms, AAA, AXB and eMC, for photon and electron beams. Basic validation, heterogeneity corrections and IMRT/VMAT dose validation for Eclipse's photon and electron algorithms have been demonstrated. The MPPG 5.a validation tests provides the opportunity for the physicist to understand the accuracy and limitations of the dose algorithm commissioned prior to clinical implementation. Comparisons between TPS calculated beam profiles and point doses were performed for three sections. An extensive set of measurements were also performed, which were compared to corresponding TPS calculations. The data collected from the dosimetric performance results obtained during the validations testing is helpful for TPS upgrade or commissioning and for routine TPS QA.

\section{Declarations}

\section{Funding}

The authors did not receive support from any organization for this submitted manuscript.

\section{Conflict of interest}


The authors have no relevant conflicts of interest to disclose.

\section{Ethical approval}

This article does not contain any studies with human participants or animals performed by any of the authors.

\section{References}

1. Shalek RJ (1977) Determination of absorbed dose in a patient irradiated by beams of $X$ or gamma rays in radiotherapy procedures. Wiley Online Library. https://doi.org/10.1118/1.594356

2. Fraass B, Doppke K, Hunt M, Kutcher G, Starkschall G, Stern R et al (1998) American Association of Physicists in Medicine Radiation Therapy Committee Task Group 53: quality assurance for clinical radiotherapy treatment planning. Med Phys 25(10):1773-1829. https://. DOI

3. IAEA (Commissioning and quality assurance of computerized planning systems for radiation treatment of cancer. IAEA technical reports series no. 430. IAEA, Vienna 2004)

4. Van Dyk J, Barnett RB, Cygler JE, Shragge PC (1993) Commissioning and quality assurance of treatment planning computers. Int J Radiat Oncol Biol Phys 26(2):261-273. DOI: 10.1016/03603016(93)90206-b

5. Smilowitz JB, Das IJ, Feygelman V, Fraass BA, Kry SF, Marshall IR et al (2015) Commissioning and QA of Treatment Planning Dose Calculations - Megavoltage Photon and Electron Beams. J Appl Clin Med Phys 16(5):14-34. https://doi.org/10.1120/jacmp.v16i5.5768. AAPM Medical Physics Practice Guideline 5.a.

6. Narayanasamy G, Saenz DL, Defoor D, Papanikolaou N, Stathakis S (2017) Dosimetric validation of Monaco treatment planning system on an Elekta VersaHD linear accelerator. J Appl Clin Med Phys 18(6):123-9. https://doi.org/10.1002/acm2.12188

7. Snyder JE, Hyer DE, Flynn RT, Boczkowski A, Wang D (2019) The commissioning and validation of Monaco treatment planning system on an Elekta VersaHD linear accelerator. J Appl Clin Med Phys 20(1):184-193. https://doi.org/10.1002/acm2.12507

8. Zhang Y, Le AH, Tian Z, Iqbal Z, Chiu T, Gu X et al (2019) Modeling Elekta VersaHD using the Varian Eclipse treatment planning system for photon beams: A single-institution experience. J Appl Clin Med Phys 20(10):33-42. https://doi.org/10.1002/acm2.12709

9. Laub WU, Merz B, Kishore M (2021) Commissioning of a Versa HD(TM) linear accelerator for three commercial treatment planning systems. J Appl Clin Med Phys 22(3):72-85.

https://doi.org/10.1002/acm2.13180

10. Xue J, Ohrt JD, Fan J, Balter P, Park JH, Kim L et al (2017) Validation of Treatment Planning Dose Calculations: Experience Working with Medical Physics Practice Guideline 5. a. International Journal of Medical Physics, Clinical Engineering and Radiation Oncology 6(1):57-72. doi:

10.4236/ijmpcero.2017.61006 
11. Jacqmin DJ, Bredfeldt JS, Frigo SP, Smilowitz JB (2017) Implementation of the validation testing in MPPG 5.a "Commissioning and QA of treatment planning dose calculations-megavoltage photon and electron beams". J Appl Clin Med Phys 18(1):115-127. https://doi.org/10.1002/acm2.12015

12. Huq MS (2006) Absorbed dose determination in external beam radiotherapy: an international code of practice for dosimetry based on standards of absorbed dose to water.

13. Archibald-Heeren B, Liu G (2016) Raystation Monte Carlo application: evaluation of electron calculations with entry obliquity. Australas Phys Eng Sci Med 39(2):441-452. https://doi.org/10.1007/s13246-016-0437-y

14. McDonald A, Williams I (2010) Australian clinical dosimetry service at ARPANSA. https://doi.org/10.1007/978-3-642-29305-4_318

15. Lehmann J, Alves A, Dunn L, Shaw M, Kenny J, Keehan S et al (2018) Dosimetric end-to-end tests in a national audit of 3D conformal radiotherapy. Physics and imaging in radiation oncology 6:5-11. https://doi.org/10.1016/j.phro.2018.03.006

16. Lye J, Kry S, Shaw M, Gibbons F, Keehan S, Lehmann J et al (2019) A comparison of IROC and ACDS on-site audits of reference and non-reference dosimetry. Med Phys 46(12):5878-5887. https://doi.org/10.1002/mp.13800

17. Nelms BE, Chan MF, Jarry G, Lemire M, Lowden J, Hampton C et al (2013) Evaluating IMRT and VMAT dose accuracy: practical examples of failure to detect systematic errors when applying a commonly used metric and action levels. Med Phys 40(11):111722. https://doi.org/10.1118/1.4826166

\section{Tables}

Table 1. Summary of the MPPG 5.a test and equipment used. 


\section{Section}

A: Basic beam validation for photon and electron

\section{Test description}

5.1 Physics module versus planning module

5.2 Reference Calibration condition checks

5.3 Planning module versus commissioning module

5.4-5.9 Basic photon

\section{Equipment}

PTW 3D Scanning water tank, PTW Semiflex chamber

PTW 3D Scanning water tank, PTW Farmer $\&$ Parallel-plate type ionization chambers

PTW 3D Scanning water tank, PTW Semiflex chamber

PTW 3D Scanning water tank, PTW

Semiflex chamber, ArcCHECK detector array

8.1-8.2 Basic electron

B: Heterogeneity corrections for photons and electrons

6.1 Ct-to-Ed calibration Electron density Phantom
curve
field

7.3-7.4 Clinical cases

7.5 External audit
6.2 Photon heterogeneity correction

8.3 Electron heterogeneity correction

7.1-7.2 PDD and output for small MLC-defined

Heterogeneous slab phantom; Farmer type ionization chamber

Heterogeneous slab phantom; Parallel-plate type ionization chamber

PTW 3D Scanning water tank, PTW E diode

C: IMRT/VMAT dose validation

$\begin{array}{ll}\text { 7.3-7.4 Clinical cases } & \begin{array}{l}\text { ArcCHECK 3D detector array, PTW 3D } \\ \text { Pinpoint ionization chamber }\end{array} \\ \text { 7.5 External audit } & \begin{array}{l}\text { Custom CIRS IMRT thorax phantom; farmer } \\ \text { type and CC13 ionization chambers }\end{array}\end{array}$


Table 2. Point dose differences for tests $5.4,5.5,5.7$ and 5.8 at 1.5 and $10 \mathrm{~cm}$ depths along the CAX between measured and calculated AAA and AXB.

Parameters \% point dose differences

\begin{tabular}{lllllllllll} 
Test & & $\mathbf{5 . 4}$ & & $\mathbf{5 . 5}$ & \multicolumn{2}{c}{$\mathbf{5 . 7}$} & \multicolumn{2}{c}{$\mathbf{5 . 8}$} & \\
\hline Energy & Depth & AAA & AXB & AAA & AXB & AAA & AXB & AAA & AXB \\
\hline $\mathbf{6} \mathbf{~ M V}$ & 1.5 & 0.28 & 0.94 & 0.97 & 0.78 & -0.13 & -0.03 & 0.79 & 0.17 \\
& 10 & 0.43 & 0.25 & 1.52 & 1.37 & 0.02 & 0.34 & & \\
\hline \multirow{2}{*}{$\mathbf{6}$ MV FFF } & 1.5 & 0.52 & 0.42 & 1.0 & 0.91 & 0.10 & 0.01 & 1.19 & 0.26 \\
& 10 & 0.68 & 0.3 & 1.62 & 1.32 & 0.92 & 0.41 & & \\
\hline $\mathbf{1 8} \mathbf{~ M V}$ & 1.5 & -3.04 & 1.06 & -0.03 & 2.0 & -1.41 & -0.71 & 1.05 & 0.94 \\
& 10 & 1.01 & 0.86 & 1.41 & 0.67 & -0.29 & -0.68 & &
\end{tabular}

Table 3. Comparisons of electron density and mean HU values of CT electron density phantom inserts in Eclipse TPS.

\begin{tabular}{lllll} 
Material & Relative electron density & CT HU & Eclipse HU & $\begin{array}{c}\text { HU } \\
\text { diff }\end{array}$ \\
\hline Lung (Inhale) & 0.19 & -813 & -801 & 12 \\
\hline Lung (Exhale) & 0.49 & -493 & -490 & 3 \\
\hline Adipose & 0.95 & -64 & -59 & 5 \\
\hline Breast & 0.98 & -22 & -20 & 2 \\
\hline Water & 1.00 & 0 & 5 & 5 \\
\hline Muscle & 1.04 & 41 & 42 & 1 \\
\hline Liver & 1.05 & 49 & 49 & 0 \\
\hline Trabecular & 1.12 & 228 & 227 & -1 \\
\hline Dense Bone $\mathbf{8 0 0} \mathbf{m g} / \mathbf{c c})$ & 1.46 & 880 & 875 & -5 \\
\hline Dense Bone $\mathbf{( 1 2 5 0} \mathbf{m g} / \mathbf{c c})$ & 1.70 & 1331 & 1331 & -4
\end{tabular}


Table 4. Point dose differences between the ratio of measured and Eclipse TPS calculated data in heterogeneous phantoms for test 6.2.

\begin{tabular}{llll|} 
Parameters & & \% point dose differences \\
Energy & Field size & AAA & AXB \\
$\mathbf{6 M V}$ & $5 \times 5$ & 1.56 & 2.09 \\
& $10 \times 10$ & 2.75 & 1.21 \\
\hline $\mathbf{6 M V}$ FFF & $5 \times 5$ & 0.85 & 0.79 \\
& $10 \times 10$ & 1.62 & -2.42 \\
$\mathbf{1 8} \mathbf{M V}$ & $5 \times 5$ & 2.11 & 0.79 \\
& $10 \times 10$ & -2.36 & 0.98 \\
\hline
\end{tabular}


Table 5. Point dose differences between the ratio of measured and Eclipse TPS calculated data in heterogeneous phantoms for tests 8.3.

\begin{tabular}{|c|c|c|c|}
\hline Paramet & & & $\%$ point dose differences \\
\hline Energy & Field size & Depth (cm) & eMC \\
\hline $6 \mathrm{MeV}$ & $10 \times 10$ & 2.5 & -5.2 \\
\hline & & 3 & -3.9 \\
\hline & & 3.5 & 5.8 \\
\hline $9 \mathrm{MeV}$ & $10 \times 10$ & 3.5 & -2.7 \\
\hline & & 4 & -6.5 \\
\hline & & 4.5 & -6.1 \\
\hline $12 \mathrm{MeV}$ & $10 \times 10$ & 6.5 & -6.9 \\
\hline & & 9 & -3.0 \\
\hline & & 10 & -4.2 \\
\hline $16 \mathrm{MeV}$ & $10 \times 10$ & 6.5 & -5.3 \\
\hline & & 7 & -5.4 \\
\hline & & 10.5 & -5.6 \\
\hline $20 \mathrm{MeV}$ & $10 \times 10$ & 6.5 & -6.0 \\
\hline & & 7 & -5.8 \\
\hline & & 12 & -3.8 \\
\hline & & 13 & -5.6 \\
\hline
\end{tabular}

Figures 

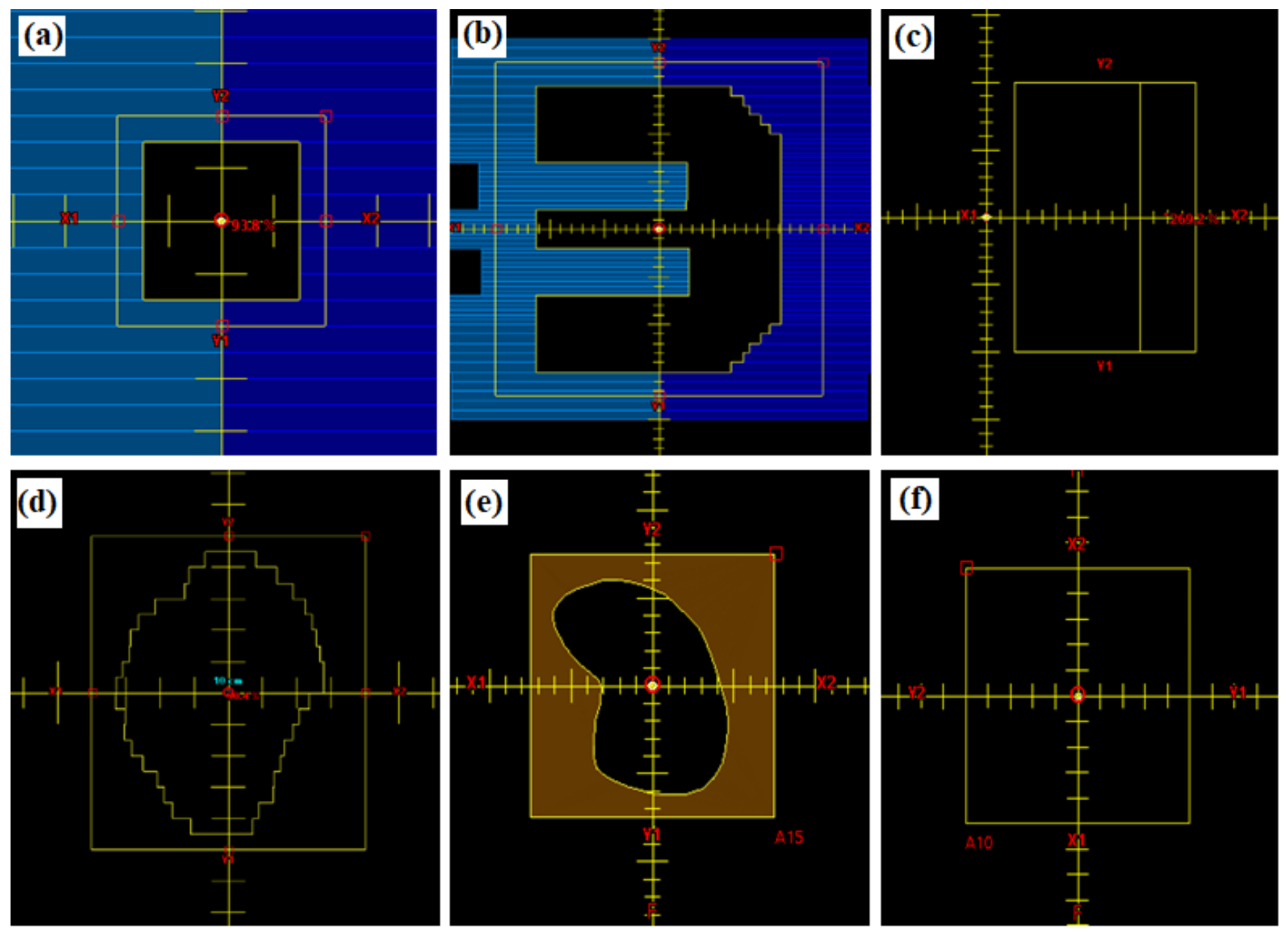

\section{Figure 1}

Fields aperture of the static MLC fields for the MPPG 5.a tests for basic beam validations section 5 (5.45.8, 8.1 , 8.2), (a) small non-SRS MLC-shape field (5.4), (b) Large MLC-shape with extensive blocking (test 5.5), (c) off-axis MLC-shape with maximum filed leaf travel (5.6), (d) Irregular MLC-shape field (5.7 \& 5.8), (e) 8.1 a custom cut-out shape in a $15 \times 15$ applicator and (f) 8.2 standard $10 \times 10$ applicator electron. 


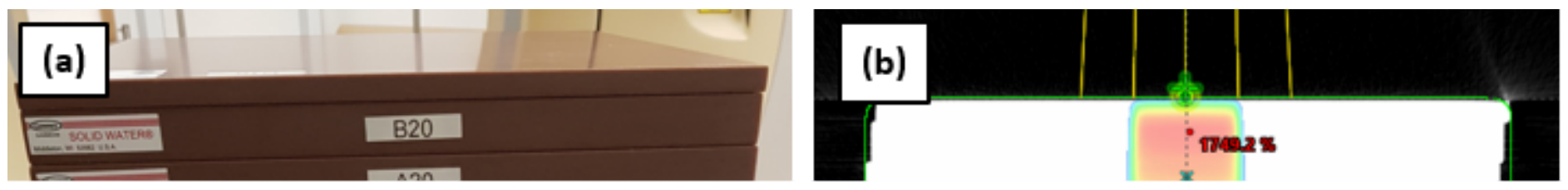

Figure 2

Heterogeneity slab geometry created for the MPPG 5.a heterogeneity tests. The phantoms are made of solid water and Styrofoam for tests 6.2 and 8.3: (a) a solid water phantom with a $13 \mathrm{~cm}$ Styrofoam heterogeneity used for photon test 6.2; (b) CT image of the phantom (a); (c) a solid water phantom with 5 $\mathrm{cm}$ Styrofoam used for test 8.3 used for electron test 8.3; (d) CT image of the phantom for test 8.3 for high energy electron. 


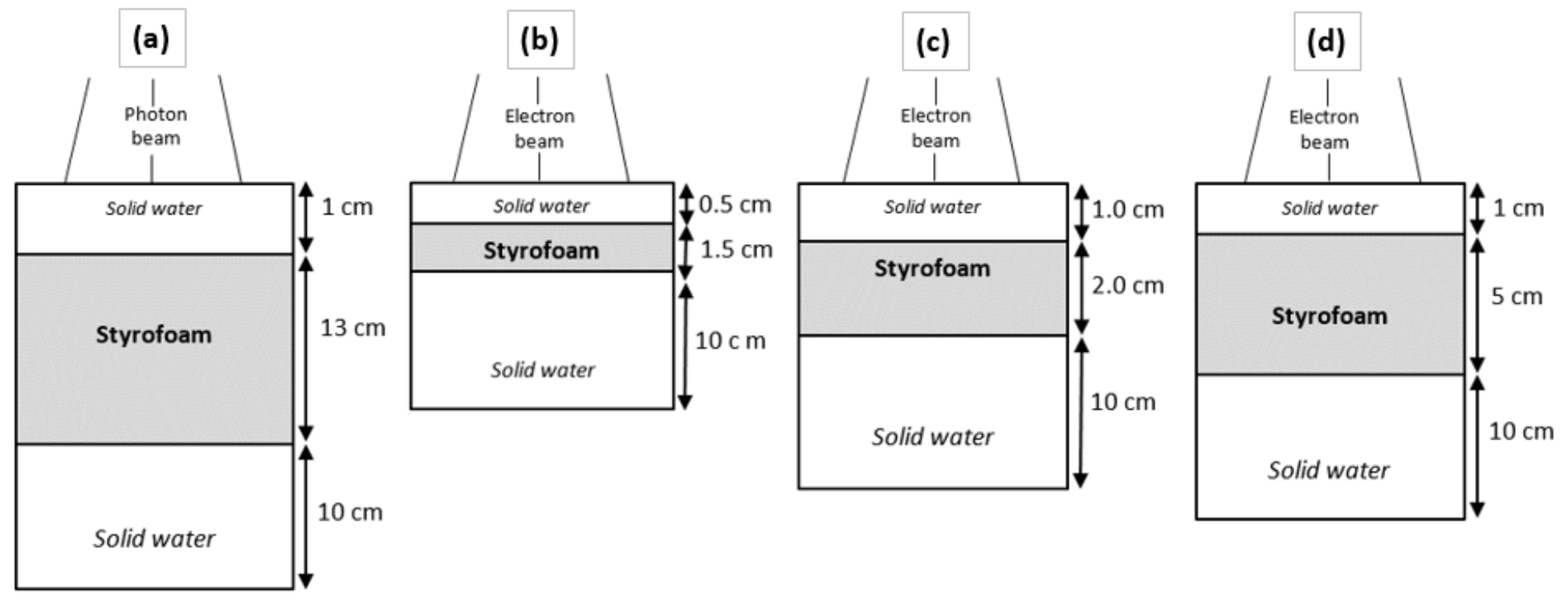

Figure 3

Schematic of phantoms geometry used photon and electron for heterogeneity testing (a) photon heterogeneity dose measurements, (b) $6 \mathrm{MeV}$ electron dose measurements; (c) $9 \mathrm{MeV}$ electron dose measurements; (d) for 12-20 MeV electron dose measurements.

(a) $6 \mathrm{MV} 40 \times 40 \mathrm{~cm}^{2}$ at $1.5 \mathrm{~cm}$ depth

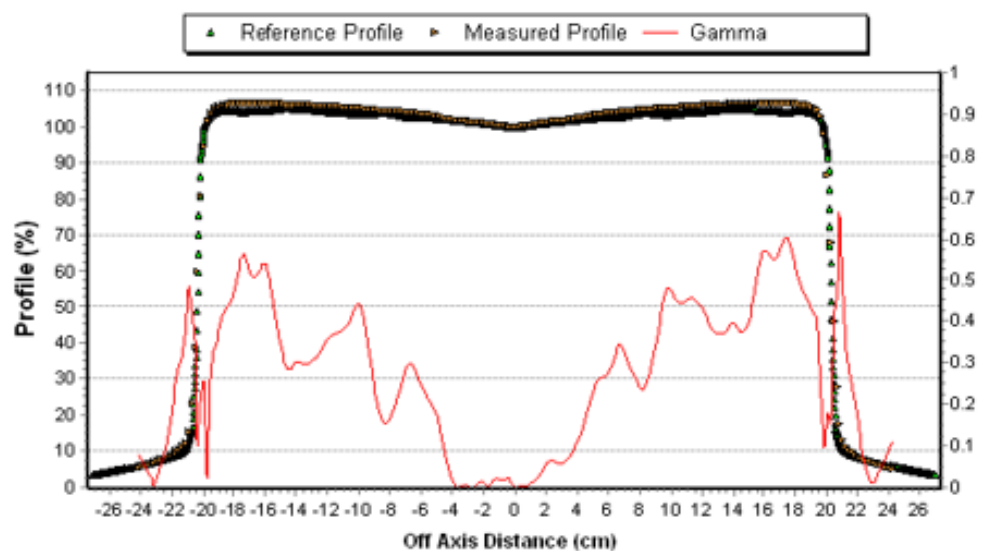

(c) 6 MV FFF $10 \times 10 \mathrm{~cm}^{2}$ at $1.5 \mathrm{~cm}$ depth

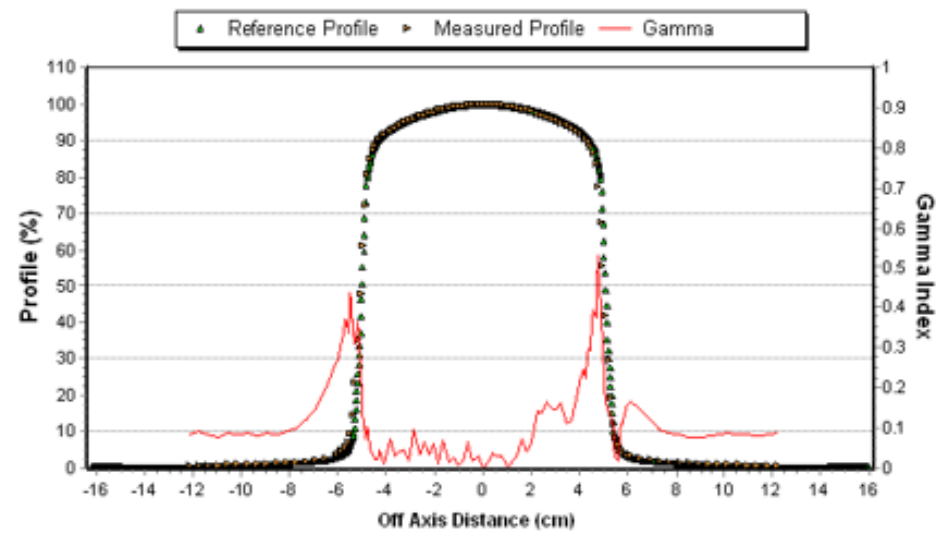

(b) $18 \mathrm{MV} 40 \times 40 \mathrm{~cm}^{2}$

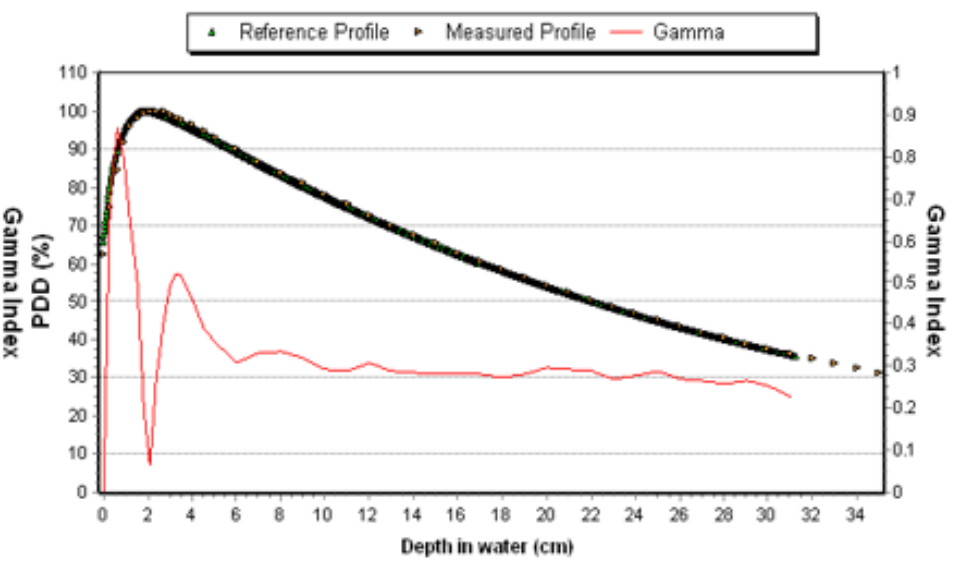

(d) Output Factor $6 \mathrm{MV}$

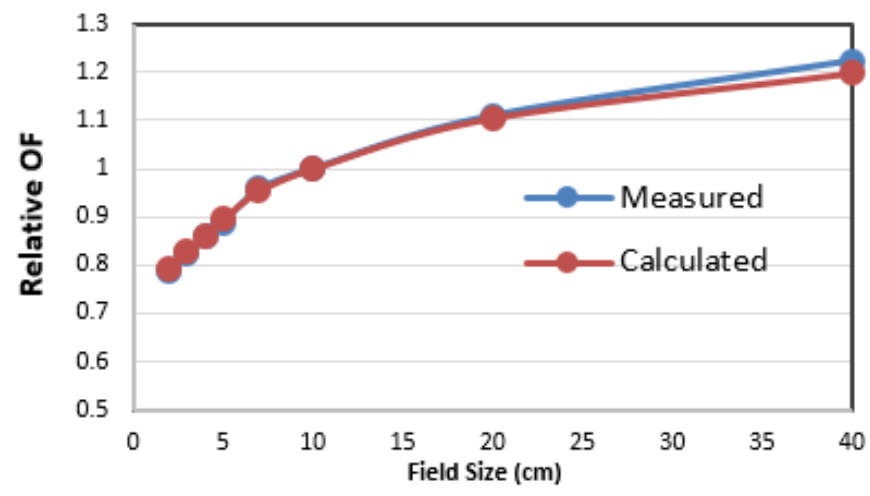


Figure 4

Comparisons between measured and calculated data for tests 5.1 and 5.3, for (a) crossplane profile of a $6 \mathrm{MV} 40 \times 40 \mathrm{~cm} 2$ field at $1.5 \mathrm{~cm}$ depth, (b) PDD of a $18 \mathrm{MV} 40 \times 40 \mathrm{~cm} 2$ (c) crossline profile of a $6 \mathrm{MV}$ Flattening filter free (FFF) $10 \times 10 \mathrm{~cm} 2$ field at $1.5 \mathrm{~cm}$ depth, and (d) output factor of a $6 \mathrm{MV}$ photon beam.
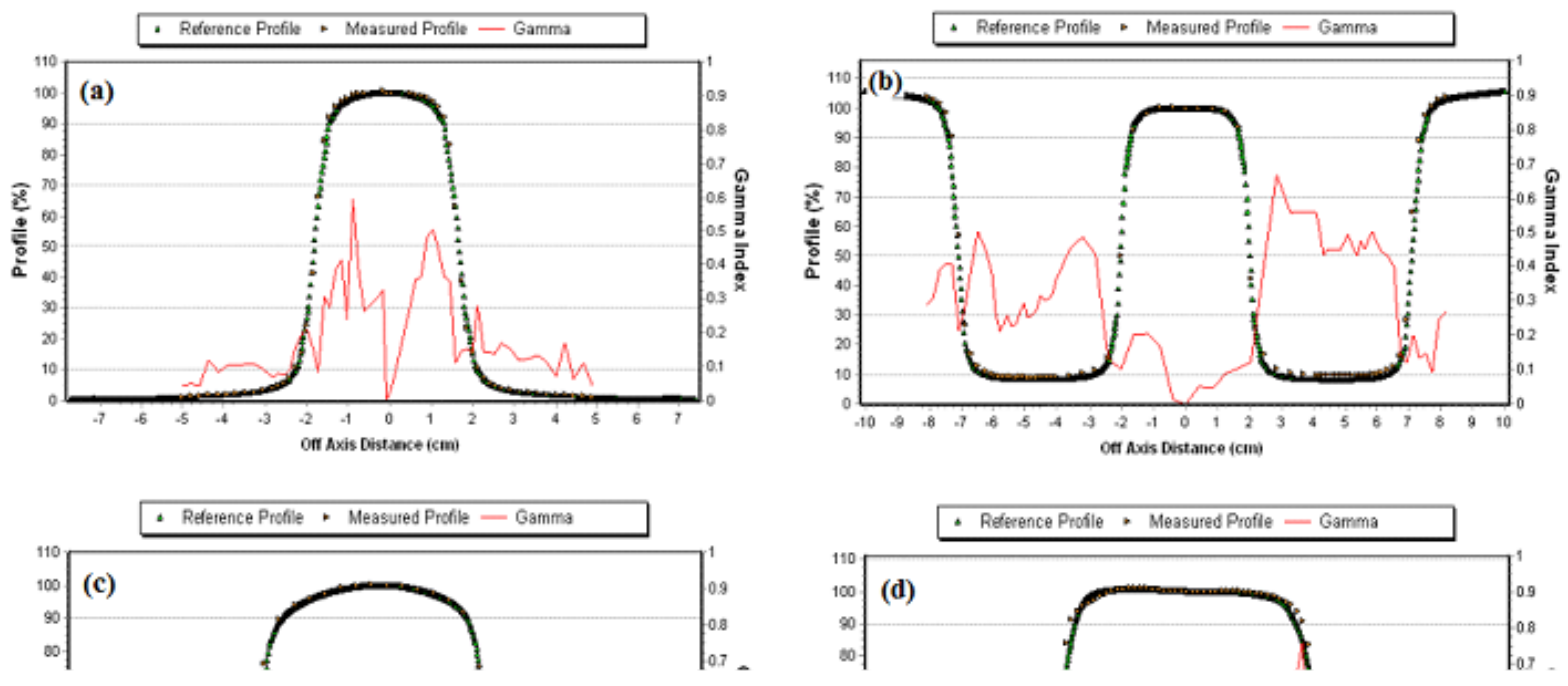

Figure 5 
Gamma analysis between measured and calculated data for tests 5.4 - 5.8, for (a) crossplane profile at $10 \mathrm{~cm}$ depth for a $6 \mathrm{MV} \mathrm{FFF}$ for test 5.4, (b) crossplane profile at $10 \mathrm{~cm}$ depth for $6 \mathrm{MV}$ for test 5.5, (c) crossline profile at $25 \mathrm{~cm}$ depth for $6 \mathrm{MV}$ for test 5.7 , (d) crossplane profile at $1.5 \mathrm{~cm}$ depth for $6 \mathrm{MV}$ FFF photon beam for test 5.8, (e) profile enhanced dynamic wedge for $18 \mathrm{MV}$ for test 5.9. (f) inline profile at the depth of $\mathrm{d} 90(2.8 \mathrm{~cm})$ for a $12 \mathrm{MeV}$ electron delivered through a cut-out shape field size for test 8.1 (g) a PDD for a $12 \mathrm{MeV}$ electron beam delivered through the standard 10x10 cone applicator at oblique beam incidence for test 8.2 .

\section{Figure 6}

Gamma analysis between measured and calculated data a PDD and crossplane profile for a $2 \times 2 \mathrm{~cm} 2$ field size. 